\title{
Compression of ECG signal using video codec technology-like scheme
}

\author{
Di-Hu Chen $* \&$ Sheng Yang
}

Department of Precision Machinery \& Instrumentation, University of Science and Technology of China, Hefei 230027, China. * Correspondence should be addressed to Di-Hu Chen(dhchen@mail.ustc.edu.cn).

\begin{abstract}
In this paper, we present a method using video codec technology to compress ECG signals. This method exploits both intra-beat and inter-beat correlations of the ECG signals to achieve high compression ratios (CR) and a low percent root mean square difference (PRD). Since ECG signals have both intra-beat and inter-beat redundancies like video signals, which have both intra-frame and inter-frame correlation, video codec technology can be used for ECG compression. In order to do this, some pre-process will be needed. The ECG signals should firstly be segmented and normalized to a sequence of beat cycles with the same length, and then these beat cycles can be treated as picture frames and compressed with video codec technology. We have used records from MITBIH arrhythmia database to evaluate our algorithm. Results show that, besides compression efficiently, this algorithm has the advantages of resolution adjustable, random access and flexibility for irregular period and QRS false detection.
\end{abstract}

Keywords: ECG compression; Video CODEC; QRS detection; Arithmetic coding

\section{INTRODUCTION}

The electrocardiogram (ECG) is an important tool for diagnosis of heart diseases. The volume of ECG data produced by modern monitoring system can be quite large over a long period of time and data compression is often needed for efficient process, store and transmit of such data. In the past, many ECG compression methods were proposed and could beclassified into three major categories [1]: a) Parameter extraction techniques. b) Transform-domain techniques.c) Direct time-domain techniques.

In this paper, we present a method for compression of ECG data using video codec technology. Since ECG signals have both intra-beat and inter-beat cor- relations like video signals with intra-frame and inter-frame correlations, video codec technology can be used for ECG compression. For ECG signals, there is a little difference, so some pre-process will be needed: ECG signals should be segmented and period normalized to a sequence of beat cycles with the same size. Then these beat cycles can be treated as 'picture frames' and compressed with a video codec.

In this work, we present a method using video codec technology to compress ECG signals. This method exploits both intra-beat and inter-beat correlations of the ECG signals to achieve high compression ratios $(\mathrm{CR})$ and a low percent root mean square difference (PRD). Although video codec technology was developed to compress video signals, it can be used to compress other signals as well, and we illustrate how video codec technology can be used to compress ECG signals. In Section II, we take abrief overview of video codec technology. Section III presents the coding algorithm. Experimental results and comparisons with other algorithm are presented in Section IV. At last, we provide conclusions.

\section{OVERVIEWOF VIDEOCODEC TECH- NOLOGY}

Representing video material in a digital form requires a large number of bits. The volume of data generated by digitizing a video signal is too large for most storage and transmission systems. This means that compression is essential for most digital video applications. Statistical analysis of video signals indicates that there is a strong correlation both between successive picture frames and within the picture elements themselves. Theoretically decorrelation of these signals can lead to bandwidth compression without significantly affecting image resolution. A video signal consists of a sequence of individual frames. Each frame may be compressed individually using an image CODEC, such as JPEG. This is described as intra-frame coding for each frame is intra coded without any reference to other frames. However, better compression performance may be achieved by exploiting the temporal redundancy in a video sequence or the similarities between successive 
ENCODER

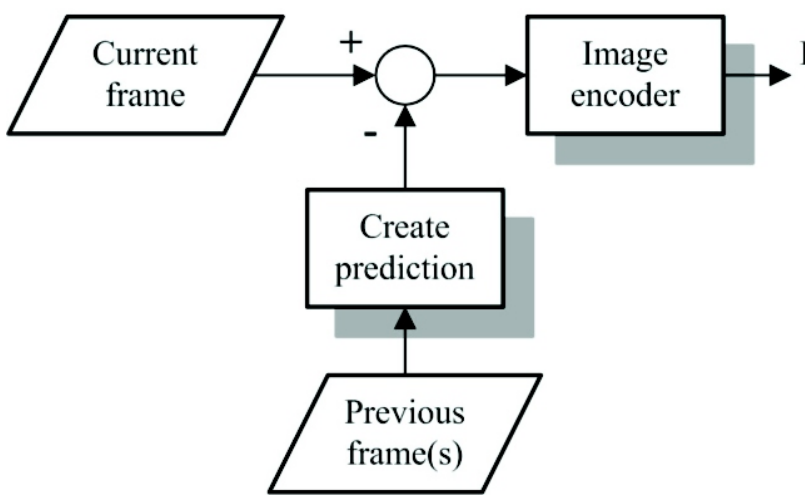

Figure 1. Video CODEC with prediction.

video frames. This may be achieved by introducing two functions: 1. Prediction: create a prediction of the current frame based on one or more previously transmitted frames. 2. Compensation: subtract the prediction from the current frame to produce a residual frame. Then the residual frame is compressed by an image CODEC. In order to decode the frame the decoder adds the prediction to the decoded residual frame. This is described as inter-frame coding for frames are coded based on some relationship with other video frames. Figure 1 shows the process above.

\section{METHOD}

\subsection{System overview}

The redundancies in ECG signals can be broadly classified into two types: The redundancies in a single ECG cycle and the redundancies across ECG cycles. These redundancies are sometimes referred to as intra-beat and inter-beat redundancies [2]. These are the same with redundancies in video signals. On the other hand, there is a little difference between video signals and ECG signals: A video signal consists of a
DECODER

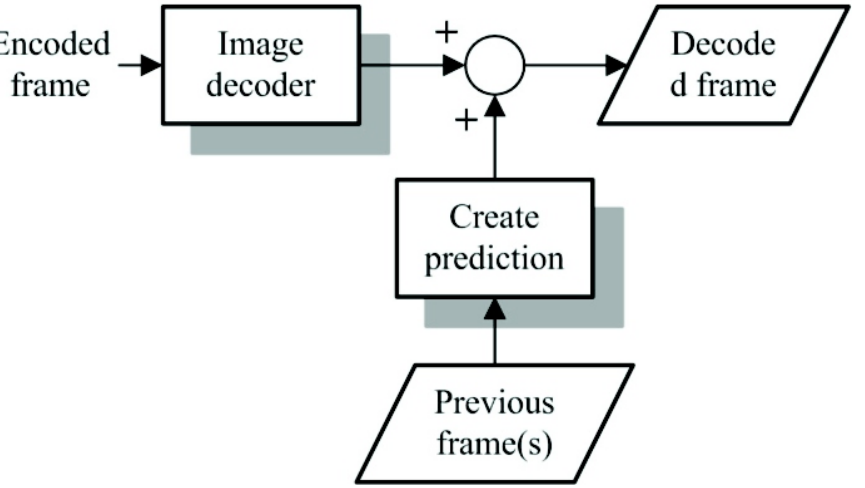

sequence of individual frames and these frames are of the same size. But for ECG signals, these 'frames' or beat cycles are jointed together, and even the sizes of them are not the same. The comparability of the ECG signals and video signals motivates us to design a novel ECG compression scheme using video codec technology, in which the scheme employs the arithmetic coding for intra-beat redundancies, and a predictor using cross correlation for inter-beat redundancies.

The functional block diagram of the proposed coding scheme is shown in Figure 2. The encoder system consists mainly four parts: segmentation, period normalization, predictor and residual coding. The proposed encoding algorithm is briefly described as follows. Since ECG signals are continuous and in order to use compress them using a video codec scheme, firstly we should segment them to a sequence of cycles, by noting that the length of each beat cycle may be varying, a period normalization process is then proceeded to ensure that the size of each beat cycle is adjusted to be the same. Initially, the counter is set to zero and we select the first cycle as the pre-

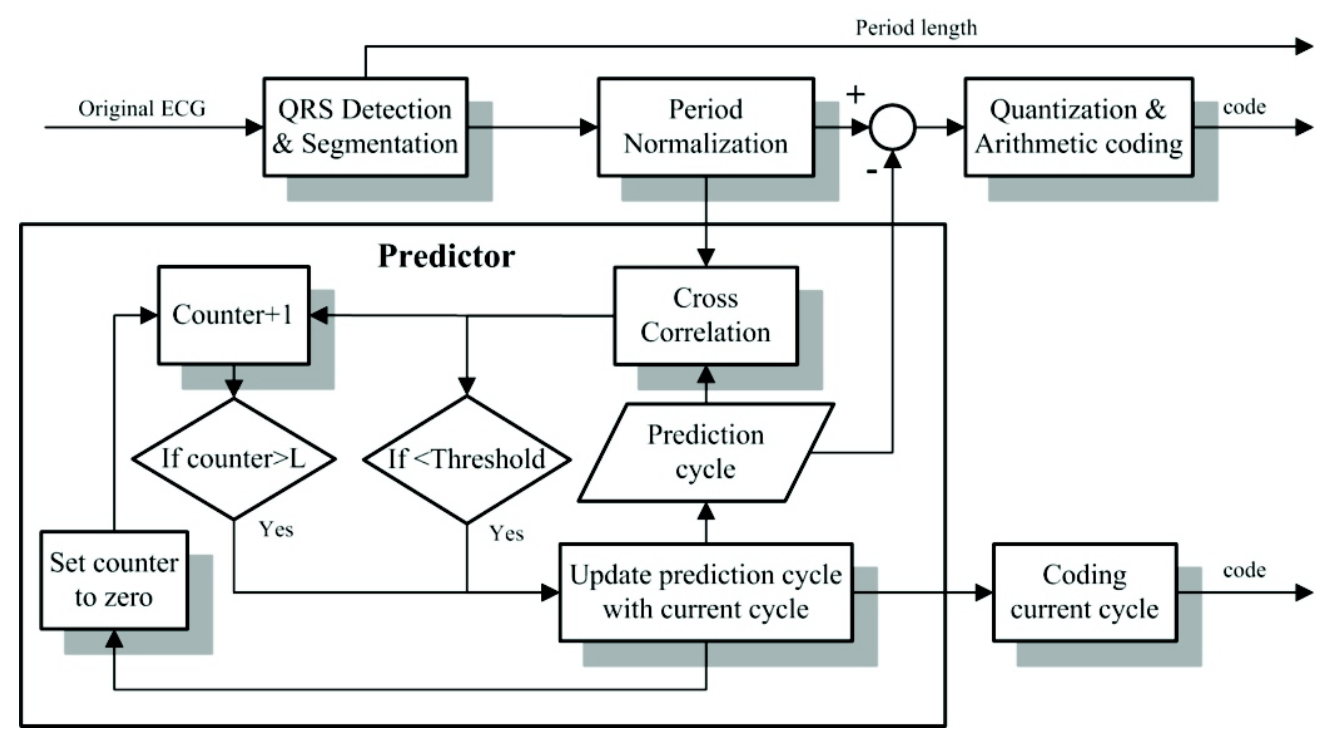

Figure 2. Functional block diagram of the encoder. 
diction cycle and compress this cycle with no prediction, then any time when there is a new cycle, the counter is added by one and the cross correlation coefficient of the new cycle and the prediction cycle is calculated. If the result is less than the threshold, which indicates that this new cycle and the prediction cycle have little similarity, or the count is larger than $L$ (used for random access), we set the counter to zero and set this new cycle as the prediction cycle and compress it with no prediction, else the prediction cycle is subtracted from this new cycle, and the residual cycle is then quantized and compressed with the arithmetic coding.

\subsection{QRS detection and segmentation}

To cut continuous ECG signals to individual beats, the peaks of QRS waves should be detected firstly to identify each heartbeat. We use a different method to do this: Let $x(i)$ denote the ECG signal, and a corresponding different signal $x^{\prime}(i)$ is given by

$$
x^{\prime}(i)=2 x(i)-x(i+n)-x(i-n)
$$

where $n$ is a small integer determined by the sampling frequency (typically a value between $0.01 f$ and $0.02 f$ is used, where $f$ is the sampling frequency). Several zero points are added to the front and the end of the ECG signals for calculation of the first and last few points of $x^{\prime}(i)$. When select proper $n$ for different sample frequency, (1) is like a band pass filter. It makes the QRS waves be amplified and the other waves be weaken. Figure 3 shows a typical ECG signal and its corresponding difference signal generating by (1). The sample frequency is $360 \mathrm{~Hz}$ with $n$ equals to 5 .

For the different signal $x^{\prime}(i)$, we can use a similar scan algorithm in [3] for the QRS detection. Results show that, our method has a higher detection rate.

After each QRS peak of heartbeat cycles is identified, the original ECG signal is cut at every QRS peak.

\subsection{Period normalization}

Since each ECG period can have a different duration, and in order to compress them using video codec tech-
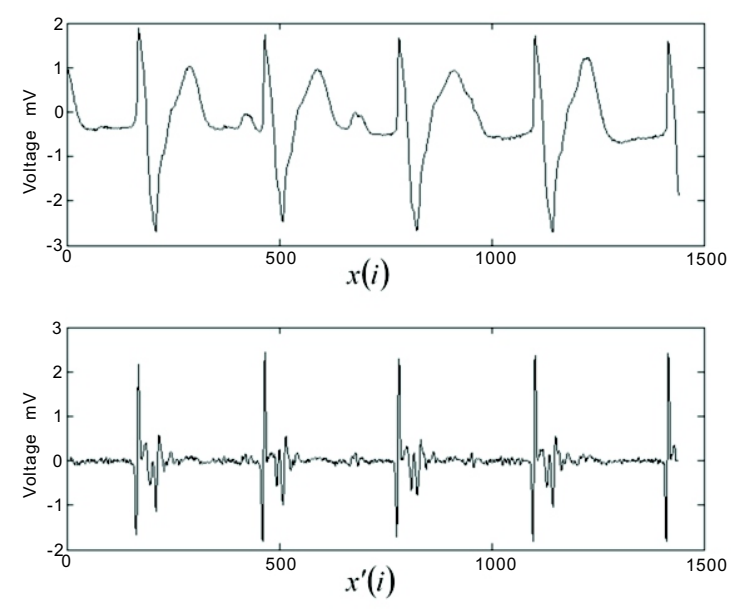

Figure 3. ECG signal and corresponding different signal. nology, we normalize each ECG period to the same length. We implement this using a method similar to the one described in [4]. Let $x_{k}=\left[x_{k}(1) x_{k}(2) \cdots x_{k}\left(N_{k}\right)\right]$ denote the $k$-th ECG cycle. Then the period-normalized ECG cycle $y_{k}=\left[y_{k}(1) y_{k}(2) \cdots y_{k}(N)\right]$ is computing using

$$
y_{k}(n)=\widetilde{x}_{k}\left(t^{\prime}\right)
$$

Where $\widetilde{x}_{k}\left(t^{\prime}\right)$ is an interpolate version of the samples $x_{k}(n)$, and $t^{\prime}=\frac{(n-1)\left(N_{k}-1\right)}{N-1}+1, N_{k}$ is the period of the $k$-th ECG cycle, and $N$ is the normalized period. We utilize cubic-spline interpolation [5] to determine $\widetilde{x}_{k}\left(t^{\prime}\right)$.

The $N$ above can be thought as the resolution, like the spatial resolution (typically $352 \times 288$ or $352 \times$ 240 pixels in MPEG-1) in a video encoder. The value of $N$ is predefined in consideration of the sample frequency and it can affect the CR and the PRD.

After period normalization, each ECG period will be with the same length like video frames with the same size. Then we can use similar video CODEC technology to compress them.

\subsection{Prediction}

In part 2 we know that, in order to exploit the similarities between successive video frames, two functions prediction and compensation are introduced. The key to this approach is the prediction function: if the prediction is accurate, the residual frame will be containing little data and will hence be compressed to a very small size by the image CODEC.

For video compression, the simplest predictor is just the previous transmitted frame. We can utilize this in ECG compression. Since successive ECG cycles are very similar all the times, we make a small change and introduce the cross correlation coefficient. Cross correlation coefficient is a standard method of estimating the degree to which two series are correlated. Consider two series $x_{i}$ and $y_{i}$ where $i=0,1,2 \ldots N-1$, the cross correlation coefficient is defined as

$$
r=\frac{\sum_{i}\left[\left(x_{i}-\bar{x}\right)\left(y_{i}-\bar{y}\right)\right]}{\sqrt{\sum_{i}\left(x_{i}-\bar{x}\right)^{2}} \sqrt{\sum_{i}\left(y_{i}-\bar{y}\right)^{2}}}
$$

Where $\bar{x}$ and $\bar{y}$ are the means of the corresponding series.

Prediction with cross correlation is shown in Figure 2. Initially we set the counter to zero. The first ECG beat cycle is set as the prediction beat cycle and compressed with no prediction. Any time when there is a new beat cycle, the counter is added by one and the cross correlation coefficient of the new beat cycle and the prediction beat cycle is calculated. If the counter is smaller than $L$ (predefined for random access) and the correlation result is higher than the threshold (typically 0.95 or more), which indicates that the prediction beat is similar with the current beat to a great extent, then we use it as the prediction 
of the current beat. Otherwise, we use the current beat to replace the prediction beat and compress it with no prediction and set the counter to zero again.

\subsection{Quantization and Coding}

The quantization stage removes less important information, such as information that does not have a significant influence on the appearance of the reconstructed ECG signals, making it possible to compress the remaining data.

In this paper, we use the arithmetic coding [6] for compression of the residual signal and the period information. An arithmetic encoder converts a sequence of data symbols in to a single fractional number. The longer the sequence of the symbols, the greater the precision required to represent the fractional number. Arithmetic coding provides a practical alternative to Huffman coding and can more closely approach the theoretical maximum compression [7].

\subsection{Coding of beat cycles}

In the video coding standard MPEG-1, each frame of video is encoded to produce a coded picture. There are three main types: I-pictures, P-pictures and Bpictures. I-pictures are intra-coded without any motion-compensated prediction. An I-picture is used as a reference for further predicted pictures. Ppictures are inter-coded using motion-compensated prediction from a reference picture. B-pictures are inter-coded using motion-compensated prediction from two reference pictures, the P-and/or I-pictures before and after the current B-picture. However, in our proposed scheme for ECG compression, we only introduced two types: I-cycles and P-cycles.

I-cycles are useful resynchronization points in the coded bit stream: because it is coded without prediction, an I-cycle may be decoded independently of any other coded cycles. This support random access by a decoder in some degree (a decoder may start decoding the bit stream at any I-cycle position). However, an I-cycle has poor compression efficiency because no prediction is used.

In MPEG-1 due to the existence of several picture types, a group of pictures (GOP) is the highest level of the hierarchy. A GOP is a series of one or more picture to assist randomly access into the picture sequence. The first coded picture in the group is an I-

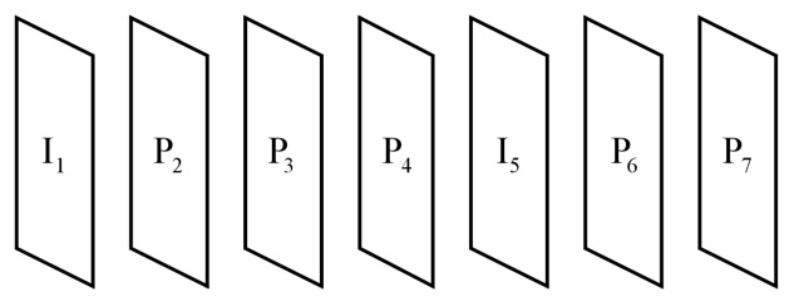

Figure 4. Group of cycles in coded bit stream. picture. It is followed by an arrangement for $\mathrm{P}$ - and B-pictures. Likewise, we introduce the group of cycles in our scheme to assist random access into the ECG data. The group of cycles length is defined as the distance between I-cycles, which is represented by parameter $L$ in Figure 2. A short group of cycles may support random access well at the cost reducing the compression ratio. Figure 4 shows a typical group of cycles.

\section{RESULT}

We used the MIT-BIH arrhythmia database to evaluate the performance of the proposed scheme. The ECG data used in our experiments are sampled at 360 $\mathrm{Hz}$ and each sample has a resolution of 12 bit per sample. Through period normalization, we have made the number of samples in each beat cycle equal 240 . Although for a typical hart rate of 75 beat per minute, 288 samples in each beat cycle will be good, but a relative small samples will increase compression ratio without obviously affecting the reconstruction quality.

We use two widely used measures, the compression ratio (CR) and the percent root mean square difference (PRD) to evaluate our scheme. The CR and PRD are defined as

$$
C R=B_{\text {ori }} / B_{c p}
$$

Where $B_{\text {ori }}$ is the total bits of the original ECG signal, $B_{c p}$ is the total bits of the ECG signal after compression.

$$
P R D=100 \times \sqrt{\sum_{i=1}^{n}\left[x_{\text {ori }}(i)-x_{\text {rec }}(i)\right]^{2} / \sum_{i=1}^{n} x_{\text {ori }}^{2}(i)}
$$

Where $\boldsymbol{x}_{\text {ori }}$ and $\boldsymbol{x}_{\text {rec }}$ are the original and the reconstructed ECG signals, and $n$ denotes the length of the signals.

Figure 5 and Figure 6 show example of ECG data from record 117 and record 119 with irregular period before and after compression.

In Table 1, the proposed method is compared with other methods in literature for record 117 and 119.

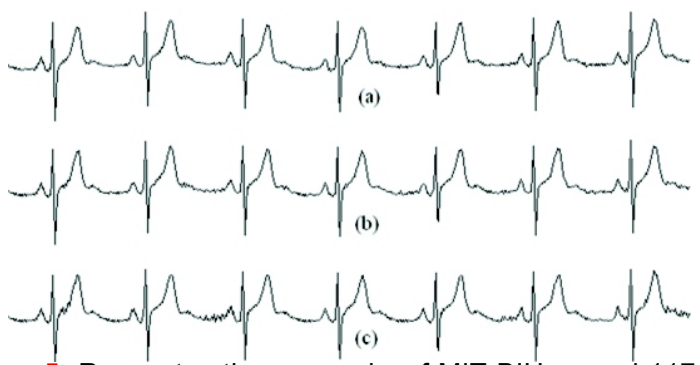

Figure 5. Reconstruction example of MIT-BIH record 117 with quantization level of $10 \mu \mathrm{V}$ and $20 \mu \mathrm{V}$ : (a) original signal of channel 1 , (b) reconstruction signal of channel 1 with quantization level of $10 \mu \mathrm{V}, \mathrm{CR}=16$ and $\mathrm{PRD}=2.87$, (c) reconstruction signal of channel 1 with quantization level of $20 \mu \mathrm{V}, \mathrm{CR}=30.79$ and $\mathrm{PRD}=5.50$. 


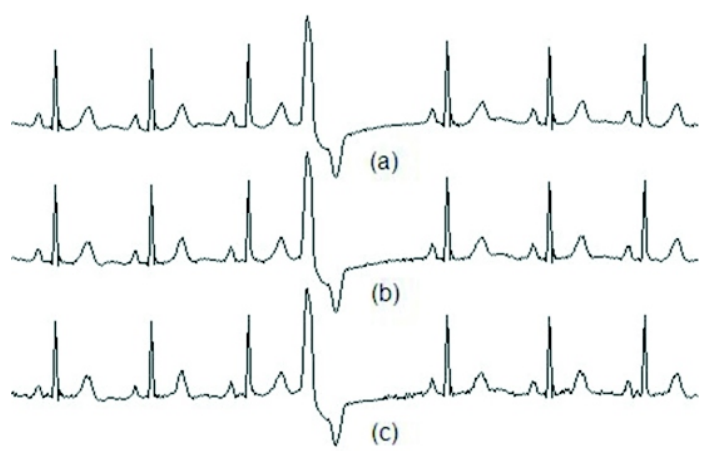

Figure 6. Reconstruction example of MIT-BIH record 119 with quantization level of $10 \mu \mathrm{V}$ and $20 \mu \mathrm{V}$ : (a) original signal of channel 1, (b) reconstruction signal of channel 1 with quantization level of $10 \mu \mathrm{V}, \mathrm{CR}=14.2$ and $\mathrm{PRD}=3.03$, (c) reconstruction signal of channel 1 with quantization level of $20 \mu \mathrm{V}, \mathrm{CR}=24.2$ and $\mathrm{PRD}=6.25$.

Table1. PRD comparison of different algorithms for record 117 and 119 .

\begin{tabular}{cccc}
\hline Algorithm & Record & CR & PRD (\%) \\
\hline Lu et. al[8] & 117 & $8: 1$ & 1.18 \\
Hilton[9] & 117 & $8: 1$ & 2.6 \\
Djohan et. al[10] & 117 & $8: 1$ & 3.9 \\
Proposed & 117 & $8.1: 1$ & 1.13 \\
Proposed & 117 & $16: 1$ & 2.87 \\
Proposed & 117 & $30.8: 1$ & 5.5 \\
Lee et.al[1] & 119 & 24 & 10.5 \\
Lu et. al[8] & 119 & 21.6 & 5.5 \\
Proposed & 119 & 14.2 & 3.03 \\
Proposed & 119 & 24.2 & 6.25 \\
\hline
\end{tabular}

\section{CONCLUSION}

The main contribution of this paper is to provide an effective and efficient ECG compression scheme using video codec technology. We have tested the performance of the proposed scheme by compressing record from the MIT-BIH arrhythmia database and compared the results with other methods. The results show that the proposed algorithm compares favorable to other methods in literature. Besides compression efficiently, the proposed algorithm benefits from characteristics of the video codec and has the following advantages: a) Resolution adjustable. By changing the length $N$ in section 3.3, we can achieve different resolution just like spatial resolution in a video codec; b) Random accessible. In coding stream of the ECG data, the I-cycles are intra-coded without any prediction, thus we can access the ECG data from every Icycle. c) Flexibility for irregular period and QRS false detection. In our scheme, the irregular periods or the QRS false detection beat cycles will be treated as the new prediction cycles and compressed with no prediction if they don't have enough similarity with the formal prediction cycle.

\section{REFERENCE}

[1] H. Lee \& K. M. Buckley. ECG data compression using cut and align beats approach and 2-D transforms. IEEE Trans-Biomed. Eng. 1999, (46):556-565.

[2]Ali Bilgin \& W. Marcellin. Compression of electrocardiogram signals using JPEG2000. IEEE Transaction on Consumer Electronics. 2003, 49(4).

[3] Engelse, W.A.H. \& Zeelenberg, C. (). A single scan algorithm for QRS detection and feature extraction. IEEE Computers in Cardiology 1979, pages 37-42.

[4] Wei, J. J., Chang, C. J., Chou, N. K.\& Jan, G. J. ECG data compression using truncated singular value decomposition. IEEE Trans. on Information Technology in Biomedicine 2001, 5:290299.

[5] T. M. Lehman, C. Gonner, \& K. Spitzer. Survey: interpolation methods in medical image processing. IEEE Trans. on Medical Imaging 1999, 18:1049-1075.

[6] James A. Storer, ed. Practical implementations of arithmetic coding. Image and textcompression, MA, 1992 pages 85-112.

[7] I. Witten, R. Neal \& J. Cleary. Arithmetic coding for data compression. Communications of the ACM 1987, 30(6).

[8] Lu, Z., D. Y. Kim, \& W. A. Pearlman. Wavelet compression of ECG signals by the set partitioning in hierarchical trees algorithm. IEEE Trans. on Biomedical Engineering 2000, 47:849856.

[9] M. L. Hilton. Wavelet and wavelet packet compression of electrocardiograms. IEEE Trans. on Biomedical Engineering 1997, 44:394-402.

[10] A. Djohan, T. Q. Nguyen \& W. J. Tompkins. ECG compression using discrete symmetric wavelet transform. Proc. of $17 \mathrm{th}$ Int. IEEE Med. Biol. Conf. 1995. 